Artículo Original. Enero-Diciembre 2019; 9:1-7. Recibido: 22/02/2019 Aceptado: 22/07/2019.

http://dx.doi.org/10.21929/abavet2019.920

\title{
Impacto económico de la mortalidad y morbilidad por enfermedades en becerras lecheras
}

\author{
Economic impact of mortality and morbidity from diseases in dairy calves
}

\section{Rocha ValdezJuan (D), Gonzalez-Avalos Ramiro (D), Avila-Cisneros Rafael (D), Peña- Revuelta Blanca 10 , Reyes-Romero Alondra}

Universidad Autónoma Agraria Antonio Narro. Torreón Coahuila México. Correspondencia: Juan Leonardo Rocha Valdez, Departamento de Ciencias Básicas, Universidad Autónoma Agraria Antonio Narro, Carretera a Santa Fe y Periférico, Torreón Coahuila México. leonardo_rv@hotmail.com, jaliscorga@gmail.com, raavci2003@yahoo.com.mx, blanca8989@hotmail.com, alondra@hotmail.com

\section{RESUMEN}

La crianza de becerras es la base del éxito de toda unidad de producción lechera. El periodo inmediatamente después del parto y los primeros días de vida son momentos críticos para ellas. La atención a los detalles durante este tiempo puede reducir las pérdidas por muertes y reducir la incidencia de enfermedades, siendo principalmente diarreas y neumonías. El objetivo del presente trabajo fue estimar el impacto económico relacionado a la mortalidad y morbilidad causada por diarrea en becerras lecheras. Se realizó un estudio observacional en una población de 510 becerras Holstein lactantes. Las variables que se consideraron para estimar el costo fueron: precio de becerra muerta, tratamiento becerra/día, días de tratamiento. Las enfermedades que se registraron para monitorear la salud de las becerras, fueron diarrea y neumonía. Se detectó un $40.4 \%$ y $8.4 \%$ de prevalencia de crías con evento de diarrea y diarrea más neumonía respectivamente. La diarrea y neumonía afectan la salud de las becerras lecheras lactantes, lo que genera pérdidas económicas por mortalidad y tratamiento al orden de \$242,000.00 en la población evaluada.

Palabras clave: desarrollo, enfermedad, manejo, morbilidad, producción.

\begin{abstract}
The raising of calves is the important basis for the success of any dairy production unit, the period immediately after calving and the first days of life are critical times for calves. Attention to detail during this time can reduce losses from deaths and reduce the incidence of diseases. The two main types of problems are diarrhea and pneumonia. The objective of this study was to evaluate the economic impact related to mortality caused by diarrhea disease in the development of dairy calves. An observational study was conducted with a population of 510 Holstein calves in lactation. The variables that were considered to evaluate the growth will be: at birth and at weaning, weight, height at the withers, daily gain and total weight gain. The diseases that were registered to monitor the health of the calves were diarrhea and pneumonia. A prevalence of $40.3 \%$ was detected for diarrhea and $4.35 \%$ for nemonies. Diseases such as diarrhea and pneumonia affect the development of lactating dairy calves, which generates economic losses due to mortality in the order of $\$ 275,000$ in the evaluated population.

Keywords: calves, diarrhea, pneumonia, development, morbidity, economic impact.
\end{abstract}




\section{INTRODUCCIÓN}

El éxito en el manejo de las becerras inicia con el primer suministro de calostro. Las becerras que reciben una adecuada cantidad de calostro, presentan altas concentraciones de inmunoglobulinas circulantes en sangre, éstas se asocian con un descenso en la morbilidad y mortalidad por ciertas enfermedades infecciosas, tales como septicemia, enteritis, diarreas y enfermedades respiratorias (Besser y Gay, 1994). Asimismo, la reducción del riesgo de morbilidad y mortalidad pre-destete, otros beneficios a largo plazo asociados a la transferencia pasiva de inmunidad, incluyen la disminución de mortalidad en el periodo posterior al destete, mejoría en la tasa de ganancia y eficiencia alimenticia, la reducción de la edad al primer parto, la mejora en la producción de leche en la primera y segunda lactancia y la reducción de desecho de vaquillas durante la primera lactancia (Faber et al., 2005).

Tasas altas de morbilidad y mortalidad en becerras recién nacidas son atribuidas a enfermedades infecciosas; las dos más frecuentes que afectan a las becerras son la diarrea y las enfermedades respiratorias. La tasa de mortalidad en becerras antes del destete es de $7.8 \%$. La diarrea y otros problemas digestivos contribuyen al $56.5 \%$ de las muertes; las enfermedades respiratorias es la segunda causa de mortalidad con $22.5 \%$ (USDA, 2010).

Los trastornos digestivos en las becerras son enfermedades frecuentes que se manifiestan con diarreas caracterizadas por heces líquidas y profusas, deshidratación, emaciación, postración y muerte (Delgado, 2000). Las enfermedades entéricas son comunes y le representa enormes pérdidas económicas a la industria de la ganadería, de la carne y leche, como resultado de la mortalidad de recién nacidos y costos de tratamiento. Es común que la diarrea neonatal sea más el resultado de una infección combinada de diferentes enteropatógenos (bacterias, virus, protozoarios), que la infección con un solo agente; siendo importante la Escherechia coli, Salmonella, Rotavirus, Clostridium, Giardia y Coronavirus. Cabe mencionar que mayores pérdidas ocurren cuando las becerras son mantenidas en confinamiento, donde la oportunidad de transmisión de los agentes causales de la diarrea se ve realizada por su acumulación en el medio ambiente (Baquero-Parrado, 2008).

Estos agentes afectan a bovinos de todas las edades, siendo las becerras recién nacidas y menores de 60 días las que presentan la enfermedad entérica en forma más manifiesta. Es importante resaltar que, aunque todos estos agentes patógenos pueden ser primarios, estudios epidemiológicos y de laboratorio han demostrado que las infecciones mixtas son más comunes que las infecciones simples, en su asociación con la presentación clínica de la enfermedad. Es por ello que en la actualidad se describe a este cuadro clínico como Complejo Diarreico Bovino (CDB), y cuando afecta a la recién nacida recibe el nombre 
de diarrea indiferenciada de la becerra. Aunque no existen estadísticas de estos trastornos en México, los patógenos gastroentéricos están asociados hasta en un 25\% con las muertes en becerras (Delgado, 2009). Las condiciones actuales están obligando al productor a ser más eficiente en la cría y desarrollo de vaquillas. Esta es un área de suma importancia, ya que lo que se haga hoy se reflejará en el futuro; el productor debe criar las vaquillas de la manera más eficiente para reducir los gastos, pero sin llegar a afectar negativamente su salud y futura productividad (Belloso, 2005; González et al. 2017).

Una reducción de gastos genera una rentabilidad privada o ganancia; siendo ésta la retribución a la disposición del productor para administrar recursos y aceptar un riesgo (Hernández et al., 2016).

Un costo es un recurso que se utiliza para obtener un objetivo específico. El sistema de contabilizar los costos de operación permite generar información acerca de las actividades productivas esenciales y administrativas que llevan a cabo las empresas en su ciclo productivo, cuantificando y clasificando los gastos según el interés de los dueños o inversionistas; como es el costo por unidad producida, el destino de los gastos y la comparación con el producto de la venta (Trejo y Floriuk, 2010).

Relacionado con los costos totales de criar una ternera desde su nacimiento a los 4 meses de edad, bajo un sistema de crianza de finca promedió los \$442.97 dólares americanos, con un rango de variación de más-menos $\$ 37.42$ dólares americanos (Elizondo y Vargas 2015).

Los animales de reemplazo se estiman dentro del 15-20 por ciento del total de los costos de producción de leche. El reemplazo de vaquillas se califica como el segundo o tercer componente más grande en costos de producción, después de la alimentación, y posiblemente en la mano de obra en la mayoría de los establos lecheros. Estos costos varían de establo a establo y pueden tener diferencias extremas, debido a los variables niveles de manejo. Los costos en vaquillas están influenciados por una variedad de situaciones. Los ranchos con altos niveles de morbilidad y de mortalidad han aumentado los costos (Heinrichs, 2001).

El objetivo del presente trabajo fue estimar el impacto, relacionado con la mortalidad causada por diarrea en becerras lecheras.

\section{MATERIAL Y MÉTODOS}

El estudio se realizó del 20 de febrero al 30 de abril del 2016, con una población de 510 animales, en un establo lechero del municipio de Torreón Coahuila, el cual se encuentra localizado en una región semidesértica del norte de México, a una altura de $1140 \mathrm{msnm}$; entre los parámetros $25^{\circ} 30^{\prime}$ y $25^{\circ} 45^{\prime}$ y los meridianos $103^{\circ} 20^{\prime}$ y $103^{\circ} 40^{\prime} \mathrm{O}$ (INEGI, 2009). 
Las variables que se consideraron para estimar el costo, fueron precio de becerra al nacimiento, tratamiento becerra/día y días de tratamiento. La diarrea y neumonía fueron las enfermedades que se registraron para monitorear la salud de los animales. El registro se realizó a partir del nacimiento, hasta los 45 días de vida. La clasificación de las crías con diarrea se realizó mediante la observación de las consistencias de las heces; heces normales corresponde a crías sanas, y becerras con heces semi-pastosas a líquidas fueron crías enfermas. En relación a la clasificación de la neumonía, las crías con secreción nasal, lagrimeo, tos y elevación de la temperatura superior a $39.5^{\circ} \mathrm{C}$, se registró cría enferma; si no presentó lo anterior se consideró una cría sana.

Las becerras recibieron una toma de 4 litros de sustituto de leche (Hi-bloom $\AA$ ), cada litro se preparó con $125 \mathrm{~g}$ de sustituto en polvo mezclado en $875 \mathrm{ml}$ de agua; se ofreció una mezcla completamente homogenizada y en una sola toma por la mañana 07:00 h a una temperatura de $39^{\circ} \mathrm{C}$; ésta se suministró hasta el destete de los animales, la cual se realizó a los 45 días de vida. El agua estuvo disponible a libre acceso a partir del segundo día de edad. Además, se complementó con concentrado iniciador con $22 \%$ de proteína cruda (PC) a libre acceso, a partir del tercer día de vida.

El análisis estadístico del crecimiento y presencia de enfermedades se realizó mediante un análisis de varianza, y la comparación de medias se realizó mediante la prueba de Tukey. Los análisis se ejecutaron utilizando el paquete estadístico de Olivares-Saenz (2012). Se empleó el nivel de significancia con un valor de $p<0.05$, para considerar diferencia estadística.

\section{RESULTADOS Y DISCUSIÓN}

En el cuadro 1, se observa la mortalidad y morbilidad en becerras lactantes ocasionado por diarrea. Estudios de salud en becerros antes del destete en Estados Unidos de Norte América, reportaron morbilidad por diarrea de $23.9 \%$ y $27.2 \%$ durante las primeras 8 semanas de vida (USDA, 2008).

La mortalidad de becerras es una importante preocupación económica y de bienestar en las granjas lecheras de todo el mundo (Mee, 2008). Como era de esperar, está creciendo el interés en caracterizar la incidencia y los factores de riesgo asociados con la mortalidad de becerras para desarrollar estrategias de reducción (Cuttance et al., 2017). 
Cuadro 1. Morbilidad y mortalidad con evento de diarrea en becerras Holstein en lactancia.

\begin{tabular}{lcccc}
\hline Variables & & Porcentaje & $\begin{array}{c}\text { Costo } \\
\text { Tratamiento } \$\end{array}$ & $\begin{array}{c}\text { Costo por } \\
\text { eventos \$ }\end{array}$ \\
\hline $\begin{array}{l}\text { Total de becerras con evento de } \\
\text { diarrea }\end{array}$ & 206 & $100 \%$ & 67.00 & $13,802.00$ \\
$\begin{array}{l}\text { Mortalidad } \\
\text { Promedio de días en tratamiento }\end{array}$ & 42 & $15.5 \%$ & $5,000.00$ & $160,000.00$ \\
Mínimo de días en tratamiento & 1 & 67.00 & 270.00 \\
Máximo de días en tratamiento & 18 & 67.00 &
\end{tabular}

Total \$

$174,342.00$

En relación a morbilidad y mortalidad de becerras lactantes ocasionadas por la diarrea y neumonía (infección mixta), (cuadro 2). Debido a su pobre capacidad inmune, en el periodo cercano al nacimiento, la cría es más vulnerable a las infecciones; además, otros elementos como el consumo insuficiente de calostro, limpieza deficiente, variaciones en el clima u otras causas que desencadenan una situación de estrés, pueden disminuir el sistema de defensa predisponiendo a la afección por enteropatógenos, y a su vez a las infecciones mixtas (Muktar et al., 2015).

Cuadro 2. Morbilidad y mortalidad con evento de diarrea más neumonía (infección mixta) en becerras Holstein en lactancia.

\begin{tabular}{lcccc}
\hline Variables & & Porcentaje & $\begin{array}{c}\text { Costo } \\
\text { Tratamiento } \$\end{array}$ & $\begin{array}{c}\text { Costo por } \\
\text { eventos } \$\end{array}$ \\
\hline $\begin{array}{l}\text { Becerras con evento de diarrea }+ \\
\text { neumonía (infección mixta) }\end{array}$ & 43 & $100 \%$ & 148.00 & $6,364.00$ \\
$\begin{array}{l}\text { Mortalidad } \\
\text { Promedio de días en tratamiento }\end{array}$ & 8.18 & $28 \%$ & 5,000 & $60,000.00$ \\
Mínimo de días en tratamiento & 1 & 148.00 & $1,210.64$ \\
Máximo de días en tratamiento & 27 & 148.00 & \\
Total \$ & & 148.00 & \\
\end{tabular}




\section{CONCLUSIONES}

Bajo las condiciones en las cuales fue desarrollado el presente estudio, permite concluir que las enfermedades diarreicas y neumónicas afectan la salud de las becerras lecheras lactantes. Se detectó un $40.4 \%$ y $8.4 \%$ de prevalencia de crías con evento de diarrea y diarrea más neumonía respectivamente, lo que genera pérdidas económicas por mortalidad y tratamiento al orden de $\$ 242,000.00$ en la población evaluada. Es importante concluir que las pérdidas económicas por mortalidad generada por las enfermedades pueden incrementarse, debido a que, de 510 becerras, 249 registraron evento de enfermedad que pudiera incrementar las tasas de mortalidad. Por lo que se sugiere realizar otras investigaciones en relación a los patógenos que ocasionan enfermedades en las becerras y la resistencia a antibióticos por parte de los mismos, y así disminuyan las pérdidas económicas.

\section{LITERATURA CITADA}

Baquero-Parrado JR. 2008. Diarrea neonatal indiferenciada: consideraciones sobre su prevención en campo. Veterinaria y Zootecnia. 2(2):59-68. ISSN 2011-5415.

Belloso VT I. 2005. Cría y desarrollo de vaquillas lecheras. Memorias de DIGAL. Día Internacional del Ganadero Lechero. Delicias, Chihuahua, México.

Besser TE, Gay CC. 1994. The importance of colostrum to the health of the neonatal calf. Department of Veterinary Microbiology and Pathology, Washington State University College of Veterinary Medicine, Pullman. Vet Clin North Am Food Anim Pract. 10(1):107117. https://doi.org/10.1016/S0749-0720(15)30591-0

Cuttance EL, Mason WA, McDermott J, Laven RA, McDougall S, Phyn CVC. 2017. Calf and replacement heifer mortality from birth until weaning in pasture-based dairy herds in New Zealand. J. Dairy Sci. 100:8347-8357. http://www.sciquest.org.nz/node/140372

Delgado GR. 2000. Diarrea de las terneras en bovinos Holstein de la Comarca Lagunera. Memorias del IX Congreso Nacional de la Sociedad Mexicana de Patólogos Veterinarios, A.C. Gómez Palacio, Dgo. Pag. 44-45.

Delgado GR. 2009. Enfermedades digestivas en las becerras lactantes. 9o Congreso Internacional de MVZ Especialistas en Bovinos. Torreón Coahuila. Pag. 1-10

Elizondo-Salazar JA, Vargas-Ramírez AM. 2015. Determinación del costo de la crianza de terneras desde el nacimiento hasta el destete en una lechería comercial especializada. Revista Nutrición Animal Tropical. 9(2):1-10. ISSN: 2215-3527. 
Faber SN, Faber NE, McCauley TC, Ax RL. 2005. Case Study: Effects of colostrum ingestion on lactational performance. Prof. Anim. Scientist. 21:420-425. https://doi.org/10.15232/S1080-7446(15)31240-7

González AR, González AJ, Peña RBP, Moreno RA, Reyes CJL. 2017. Análisis del costo de alimentación y desarrollo de becerras de reemplazo lactantes. Revista Mexicana de Agronegocios. XXI (40):561-569. ISSN1405-9282.

https://www.redalyc.org/jatsRepo/141/14152127005/index.html

Heinrichs J. 2001. Análisis económico para programas eficientes de reemplazo de vaquillas. Memorias de DIGAL. Día internacional de ganado lechero. Delicias, Chihuahua México . https://extensión.psu.edu>abalisis-econo

Hernández MJ, Rebollar RA, Mondragón A, Guzmán SE, Rebollar RS. 2016. Costos y competitividad en la producción de bovinos de carne en el sur del Estado de México. Investigación y Ciencia. No 69:13-20. ISSN: 1665-4412.

Instituto Nacional de Estadística y Geografía (INEGI). 2009 Prontuario de información geográfica municipal de los Estados Unidos Mexicanos. Francisco I. Madero, Coahuila de Zaragoza. Clave geoestadística 05009. Disponible en: http://www3.inegi.org.mx/rnm/index.php/catalog/20.

Mee JF. 2008. Prevalence and risk factors for dystocia in dairy cattle: A review. Vet. J. 176:93-101. doi: 10.1016/j.tvjl.2007.12.032.

Muktar Y, Gezhagne M, Biruk T, Dinaol B. 2015. A review on major bacterial causes of calf diarrhea and its diagnostic method. J. Vet. Med. Anim. Health. 7(5):173-185. doi: 10.5897/JVMAH2014. 0351.Article Number: 31D529A52229.

ISSN 2141-2529

Olivares-Sáenz E. 2012. Paquete de diseños experimentales. FAUANL. Versión 1.1. Facultad de Agronomía Universidad Autónoma de Nuevo León. Marín, N. L., México.

Trejo GE, Floriuk GF. 2010. Costos de producción del becerro. Boletín informativo. Fideicomisos Instituidos en Relación con la Agricultura. 9. www.fira.gob.mx/InfEspDtoXML/abrirArchivo.jsp?abreArc=3678

USDA. 2008. Dairy 2007, Part III: Reference of dairy cattle health and management practices in the United States, 2007. USDA-APHIS-VS, CEAH, Fort Collins, CO. \#N482.0908.

USDA-NAHMS. 2010. Dairy 2007, Heifer calf health and management. Practices on U.S. Dairy operations. USDA: APHIS: VS, CEAH. Fort Collins, CO. \#550.0110. 